\title{
Editorial: Innovations in thoracic surgery
}

\section{A. Maier}

Dear Readers,

In this issue of European Surgery, innovations of thoracic surgery in the treatment of different thoracic malignancies will be presented from the surgical point of view.

One of the biggest challenges in thoracic surgery of the first decade of the 21 st century was a shift of paradigm in the treatment concept of lung cancer. Whereas up to that time thoracotomy had been the undisputed surgical approach for oncological indications, VATS was emerging and has now become a routine for subsegmental resections. The acceptance of VATS in lobectomy did not come by easily. Due to the unavoidable impairment of intercostal nerves also when performing VATS, the reduction of pain if compared to thoracotomy is less pronounced than in e.g. laparoscopic procedures versus those done over routine laparotomy. Some limitation of oncologically necessary procedures such as complete mediastinal lymphadenectomy seemed the crucial drawback of VATS. To date, the thoracic society defines VATS lobectomy as a procedure identical to the open procedure, but without the trauma to the chest wall inherent to thoracotomy. On the international scale, VATS lobectomy has been performed for more than 10 years by a rather limited number of surgeons and institutions. Indications, patients' selection, surgical technique and outcome, will be presented by the thoracic surgery group of the Medical University of Innsbruck, where the first VATS-lobectomy program of Austria started in 2009, with very encouraging results.

Parenchyma-sparing procedures are routinely applied in lung surgery, since many patients with lung cancer present with severe emphysema mostly caused by tobacco consumption. In the most severe cases, however, parenchymal resections are not feasible at all, because any loss of lung surface would be fraught with the risk of respiratory insufficiency. In these patients external beam irradiation and chemotherapy are the standard treatment options. Nevertheless, alternative therapeutic methods in case of functional nonresectability and poor performance status have been sought. The interdisciplinary group of thoracic surgery and radiooncology of the Medical University of Graz studied an intra-operative radiotherapy protocol (IORT) with complete lymphadenectomy as alternative treatment in functionally nonresectable non-small cell lung cancer patients.

Locally or loco-regionally advanced lung cancer presents a maximum therapeutic challenge. Multimodal treatment including induction chemo- or radiotherapy, surgery and postoperative nonsurgical oncological treatment has become standard procedure for advanced lung cancer. The sulcus superior tumours count among the best examples of advances of multidisciplinary approach: It allows for resection and definitive cure in a type of nonsmall cell carcinoma of the lung that has long defied therapeutic success. The thoracic surgery group of the Medical University of Vienna, one of the high volume centres of Europe dealing with this special type of lung cancer patients, presents the results after a multidisciplinary approach of sulcus superior carcinoma.

Photodynamic therapy (PDT), based upon administration of a photosensitizer that accumulates in tumour cells and subsequent application of red laser light to the tumour surface has become an important tool for the treatment of early-stage malignant lesions in the tracheobronchial system and in the oesophagus. The method that has been in clinical use for about 25 years causes selective destruction of tumour cells within the range of the laser beam and seems to initiate immunological processes directed against residual tumour cells. More recently, PDT has shown to be very efficient in the palliative setting of lung- and oesophageal cancer as well as malignant mesothelioma. The thoracic surgery group of the Medical University of Graz, one of the biggest European centres for PDT, gives an overview of indications and results of PDT of thoracic malignancies.

Alfred Maier President of the Austrian Society for Thoracic and Cardiovascular Surgery

\section{Conflict of interest}

The author declares that there is no conflict of interest. 